\title{
CONTRIBUCION AL ESTUDIO DE LAS CUBETAS SUPRALITORALES (TOSSA, COSTA BRAVA).
}

\author{
Jordi Catalan y Enric Ballesteros \\ Departament d'Ecologia. Facultar de Biologia. Universitat de Barcelona \\ Palabras clave: Rock pools biology, Costa Brava (Spain) rock pools.
}

\begin{abstract}
CONTRIBUTION TO THE STUDY OF ROCK POOLS AT TOSSA, COSTA BRAVA (SPAIN)

Physical, chemical and biological conditions were studied during one rainy day (23-4-1981) in ten rock pools from Tossa (Costa Brava). The strong changes of the water conductivity $\left(80,000\right.$ to $\left.2,500 \mu \mathrm{S} . \mathrm{cm}^{\mathrm{i}}\right)$ in a short time show the osmotic regulation capacity that the organisms living there must have.

Composition and concentration of neuston, plankton and benthos are also given from other visits to the pools.

Finally, some considerations are made about naturalistic, physiological and ecological aspects of the rocks pools.
\end{abstract}

\section{INTRODUCCION}

Las cubetas supralitorales constituyen un biotopo sometido a cambios drásticos y situaciones extremas en sus condiciones físico-químicas. Jas especies que en ellos habitan deben estar adaptadas a resistir grandes fluctuaciones, de forma que la composición florística y faunística de las cubetas resulta muy particular (Droop, 1753; Bourrelly, 1958). Empieza a ser notable el número de trabajos sobre ellas, sin embargo, no existe todavía un conocimiento exhaustivo de sus composición específica ni, sobre todo, de su fisiología.

En las costas mediterráneas son pioneros los estudios de Feldman (1937), que se ocupan exclusivamente de la flora. Margalef (1949) realizó una serie de medidas de clorinidad, temperatura, $\mathrm{pH}$ y concentración de oxígenoen las cubetas supralitorales de Blanes (Costa Brava), presentando una tipificación basada en esos parámetros y en los organismos presentes. Posteriormente, Vacelet $(1959,1969)$ efectuó e n Marsella diversos estudios sobre aspectos físico-químicos y del papel de los organismos en los ciclos del nitrógeno y del fósforo. Por otra parte, Pérès y Picard (1964)

Limnética 1: 43-50(1984)

(C) Asociación Española de Limnología, Madrid. Spain realizaron una síntesis bionómica de estas cubetas, y más recientemente, Chrétiennot (1971) estudió la composición fitoplanctónica de las cubetas supralitorales, también en la región de Marsella. Por último, Ballesteros $(1982,1983)$ ha realizado algunos estudios de carácter sistemático en el litoral de Tossa y en las islas Medas.

En este trabajo presentamos algunos datos obtenidos en observaciones aisladas que los autores realizaron en distintas visitas a cubetas supralitorales, situadas en Tossa, durante los años 1980 a 1983.

\section{METODOLOGIA}

Las cubetas estudiadas se encuentran cerca de la zona denominada "Es Recó" y se asientan sobre sustrato granítico. El 23 de abril de 1981, en condiciones climatológicas desfavorables y bajo una fuerte lluvia, se tomaron a las diez de la mañana medidas de diversos parámetros físico-químicos (temperatura, conductividad, $\mathrm{pH}$, nitritos, nitratos y fosfatos), siguiéndose la temperatura y el $\mathrm{pH}$ desde las 6 a las 22 horas. A fin de mostrar la variabilidad existente y las 
fluctuaciones en distintas cubetas, se escogieron diez, cuyas características permiten incluirlas en el tipo D de Margalef (1949), situadas en el horizonte de Lecanora belicopis y Caloplaca interfulgens (Ballesteros, 1982). Al mismo tiempo, en esa visita se estudió la composición florística de las principales especies animales.

En diciembre de 1980, febrero de 1982 y febrero de 1983, se efectuaron otras visitas, recogiéndose muestras para el estudio de los organismos y la concentración pigmentaria del neuston y del plancton.

La temperatura se midió con un termómetro tras coger agua a distintas profundidades La conductividad se evaluó mediante un conductímetro YSIModelo 33. Para el pH se utilizó un método colorimétrico. La determinación de nitratos, nitritos y fosfatos se realizó siguiendo la metodología descrita en Margalef et al. (1976).Las medidas de pigmentos se efectuaron en un espectrofotómetro de doble haz (Perkin-Elmer, 124), tras ser extraídos con metanol; estimándose los valores de clorofila según la fórmula de Talling y Driver (1963)

Para la determinación de los organismos se utilizaron principalmente muestras in tive, examinándose directamente al microscopio óptico. Para las valoraciones cuantitativas las muestras se fijaban con lugol y el contaje se realizaba, tras sedimentación, en un microscopio invertido (Vollenweider, 1969). Para el estudio de lá película superficial se empleó el método de Naumann (en Schwoerbel, 1975).

\section{RESULTADOS}

1.- Variabilidad de las características físicoquímicas (23-4-1981).

En la tabla I se recogen las características de las 10 cubetas estudiadas y los valores para los parámetros físico-químicos.

La oscilación de la temperatura aérea fue solamente de $4,2^{\circ} \mathrm{C}$ (t. $\max .14,0^{\circ} \mathrm{C}$ a las $14 \mathrm{~h}$. t. min. $9,8^{\circ} \mathrm{C}$ a las $6 \mathrm{~h}$ ), debido a las especiales condiciones climáticas. Estuvo lloviendo todo el día. En las cubetas la temperatura osciló muy poco $\left(2{ }^{\circ} \mathrm{C}\right)$, con apenas diferencias, unas pocas décimas, entre el fondo y la superficie. Estas variaciones carecen de importancia, sin embargo, cabe esperar que, en días de insolación normal para esa época del año, las oscilaciones de la temperatura del agua sean notablemente mayores.

La fuerte lluvia que empezó poco antes de las $6 \mathrm{~h}$., momento en que se inició la recogida de muestras, diluyó en gran medida la totalidad del agua en las cubetas pequeñas y las capas superficiales de las de mayor volumen, como se deduce de las medidas de conductividad realizadas a las $10 \mathrm{~h}$. (Tabla I). En el fondo de las cubetas mayores, la conductividad se mantenía elevada, con valores muy superiores a los del mar. Un cambio de la concentración de sales en el medio en tan poco tiempo supone una capacidad osmorreguladora notable en los organismos que en el se encuentran.

Las medidas de $\mathrm{pH}$ se realizaron en superficie y las oscilaciones fueron relativamente elevadas (de 0,4 a 1,2 unidades) (Tabla ll), como resultado de la

Tabla 1 - Algunas caracteristicas de las cubetas supraliturales estudiadas el 2 ide Abril de 1081 Conductividaden 1 S/cm Nitratos, nitratos y fosfaros en $\mu$ rat $/ 1$ Se dil la diferencia entre las temperaturas máxima y mínima del agua; así como los valores extremos de pH Some characteristics of the rocks pools studied in April of 1981 Conductivity in $\mu \mathrm{S} / \mathrm{cm}$ Nitrite, nitrate and ortho-phospate in $\mu$-at $/ 1$ Difference between high and low temperatures of water and rxtrem values of $\mathrm{pH}$ are given

Volumen (1) Profundidad $(\mathrm{cm})$

Osc térmica $\left({ }^{\circ} \mathrm{C}\right)$

Oscilación pH

Conductividad sup

Conductividad fondo

Nitritos

Nitratos

Fosfatos

Cel./ml superficie

Cel. $/ \mathrm{ml}$ fondo

$\begin{array}{cccc}1 & 2 & 3 & 4 \\ 38 & 450 & 22 & 110 \\ 15 & 21 & 12 & 10 \\ 2,2 & 2 & 2 & 2,2 \\ 8,4-8,8 & 8,6-9,2 & 8,2-8,8 & 8,4-9,0 \\ 3130 & 25430 & 5990 & 8169 \\ 2038 & 54713 & 29953 & 35944 \\ 36,15 & 0,96 & 6,63 & 26,87 \\ 117,13 & 15,19 & 145,68 & 32,42 \\ 1,60 & 1,95 & 23,5 & 71 \\ 3779 & 10858 & 1219 & 123033 \\ 5997 & 3988 & 7835 & 26980\end{array}$

$\begin{array}{ccc}5 & 6 & 7 \\ 113 & 13 & 200 \\ 13 & 10 & 20 \\ 2,8 & 2,2 & 2,5 \\ 8,4-8,8 & 8,2-9 & 8.2-8,4 \\ 7436 & 10498 & 67040 \\ 35811 & 811125 & 82672 \\ 13,5 & 11,69 & 0,24 \\ 103,5 & 5,19 & 1,76 \\ 62 & 110 & 1,3 \\ 9961 & 192862 & 3385 \\ 1400 & 455818 & 236040\end{array}$

$\begin{array}{ccc}8 & 9 & 10 \\ 277 & 5 & 7 \\ 16 & 7,5 & 9 \\ 2,2 & 2,2 & 2 \\ 8,2-8,4 & 8,2-9,4 & 8,4-9,4 \\ 11814 & 4641 & 2549 \\ 31498 & 4641 & 7222 \\ 2,41 & 2,05 & 29,0 \\ 5,79 & & \\ 18 & 140 & 53 \\ 4199 & 768068 & 9535 \\ 77317 & 675559 & 35556\end{array}$


Tabla II. - Variación del pH a lo largo del día 23 de Abril de 1981 en las distintas cubetas Daily pH variation during 23th April of 1981

\begin{tabular}{cccc} 
& \multicolumn{3}{c}{ Horas } \\
Cubetas & 6 & 14 & 22 \\
I & 8,4 & 8,8 & 8,4 \\
2 & 8,6 & 9,0 & 9,2 \\
3 & 8,2 & 8,8 & 8,4 \\
4 & 8,4 & 9,0 & 8,8 \\
5 & 8,4 & 8,8 & 8,4 \\
6 & 8,2 & 9,0 & 8,8 \\
7 & 8,2 & 8,4 & 8,2 \\
8 & 8,2 & 8,4 & 8,2 \\
9 & 8,2 & 9,4 & 8,6 \\
10 & 8,4 & 9,4 & 9,4
\end{tabular}

actividad fotosintética y respiratoria del elevado número de organismos. El valor máximo se presentó, como era de esperar, después del mediodía, cuando los efectos de la fotosíntesis son más evidentes.

En relación con los nutrientes para el crecimiento algal, son de destacar las elevadas concentraciones de nitratos, nitriros y fosfatos, qiie superan las previsiones de Margalef (1949). Los fosfatos se encuentran en concentraciones de 10 a 1000 veces las del mar. I.os nitratos y nitriros aunque comparativamente no tan elevados, también son exagerados. Las causas son las mismas qiie las citadas por Margalef (1949) en Blanes, también centro turístico, donde hay influencia de visitantes racionales que muestran afición a estos charcos, para hacer sus necesidades en ellos o en sus bordes" (Margalef, 1949). No obstante, es muy probable que concentraciones semejantes se den en las cubetas de muchas islas del litoral, en las que el guano de las gavatas (Lama argentatus) supone un importante aporte de nutrientes, juntocon los fragmentos de algas y fanerógamas marinas arrojadas por el mar.

\section{2.- Caracteristicas biológicas.}

Diferenciamos en la exposición tres comunidades neuston, plancton y bentos.

\subsection{Neuston.}

Consideramos como organismos pertenecientes al neuston a todos aquellos asociados a la película superficial. En la Tabla III se indican los organismos encontrados en el neuston de las cubetas estudiadas en diciembre de 1980.

El número de bacterias en el neuston resulta muy elevado, por contaje directo tras tinción con violeta de genciana, se estimo del orden de $10^{8}$ células por centímetro cuadrado. Predominaban con diferencia los tipos bacilares, apareciendo también algunas formas espiraladas.

En el fitoneuston cabe destacar Brachiomonas submarina, el cual llega a formar poblaciones suficientemente densas, como para que las células se encajen unas con otras, formando, debido a una intensa producción de material extracelular, una espuma superficial de varios milímetros de grosor. Los "blooms" de B. submarina aparecen preferentemente en otoño y primavera, en situaciones de elevadas cantidades de nutrientes y condiciones metereológicas estables. En febrero de 1983, estimamos coricentraciones de $2 \times 10^{6}$ células por milímetros. En la Tabla IV (ofrecemos distintos valores de cantidad de clorofila en estos "blooms" neustónicos.

En algunas cubetas la espuma superficial está formada por especies de Chlamydomonas, las cuales aparecen en ocasiones mezcladas con Brachiomonas. El resto de componentes fitoneustónicos son diatomeas y algunas cianofíceas, todas ellas componentes muy minoritarios.

El zooneuston está integrado fundamentalmente por ciliados, especialmente hipotricos, y pequeños flagelados (Bodo. Oicomonas). La mayoría de ciliados son formas con aplanamiento dorsoventral del cuerpo, moviéndose con la cara ventral dirigida hacia la superficie (Rbopalophrya. Trachelopbyllum. Chilodonella e hipotricos). En Chilodonella y Euplotes se observó la diferenciación de dos tamaños para una misma cubeta, uno aproximadamente el doble del otro (nosabríamos precisar si se trataba de especies distintas, la observación in án'o no nos di6 indicios para ello). La forma de mayor tamaño se alimentaba de fitoplancton, mientras que la pequeña se limitaba a bacterias y quizás detritos.

En una de las cubetas se observaron individuos del coleóptero Ochtbebius (Callobius) quadricollis, que recorría la superficie con la parte del cuerpo orientada hacia arriba. Probablemente obtenía así su alimento, ingeriendo el fironeuston.

En mayo de 1983, se observaron densas poblaciones

Tabla IV Clorofila a $\mathrm{mg} / \mathrm{m}$ - en tres cuberas con espumas superficiales de Brabimsnue smbmarima (Febrero. 1982)

Chlorophyll a concentration $\mathrm{mg} / \mathrm{m}$ in three rock pools with surface blowms of Brabiommat whmarina (February, 1982)

$\begin{array}{lccc} & 1 & 7 & 3 \\ \text { NEUSTON } & 142,25 & 238,8 & 147,69 \\ \text { PLANCTON } & 108,42 & - & - \\ \text { TOTAL } & 250,67 & - & -\end{array}$


de un colémbolo (fam. Hypogastruridae), formando grandes agrupaciones sobre la película superficial. A pesar de que en la literatura se cita que los colémbolos de esta familia están presentes sólo accidentalmente en el agua, los hemos ido encontrando repetidamente en las cubetas.

\subsection{Plancton.}

Los organismos planctónicos identificados en las diez cubetas estudiadas en abril de 1981 se relacionaban en la tabla $\mathrm{V}$, indicándose su abundancia.

En el fitoplancton son especialmente abundantes diversas Cblamydomonas, Pyramimonas. Tetraselmis fontiana, y sobre todo Brachiomonas submarina. Esta última especie presenta diferentes morfologías (fig.1) que, ocasionalmente, pueden coexistir en la misma cubeta. Se observa un gradiente contínuo entre las formas extremas, algunas de las cuales se aproximan y son difícilmente separables, a B. uestiana.e incluso a $B$. gracilis (ver Huber-Pestalozzi, 1961). Los estadios de división entre B. submarina y B. uestiana son más fácilmente discernibles, puesto que en la primera las autósporas están contenidas en una envoltura sin brazos laterales (fig. 1), mientras que la envoltura de la segunda sí los posee. En nuestro caso, solamente hemos encontrado formas de división de B. submarina.

En la Tabla V puede observarse que no es rara la presencia de una especie de Spbaerellopsis. En la bibliografía a nuestro alcance no hemos encontrado ninguna especie que coincida con nuestro organismo. Damos su descripción: Posee un cloroplasto tipo Euchlamydomonas, con un pirenoide subbasal y un estigma redondo medio-anterior. La célula posee un contorno variable que va de formas ovaladas a ligeramente piriformes, con una papila redondeada

Tabla III. - Organismos asociados a la película superficial del agua Se indica abundancia relativa desde (+) raro, a (5) muy abundante Species associated with water surface in the rock pools. Relative abundance, from ( + ) uncommon, to ( 5 ) very abundant

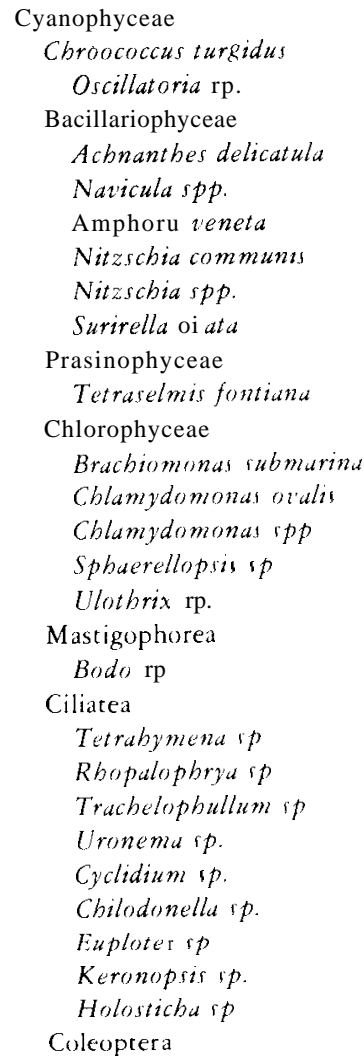

oleoprera

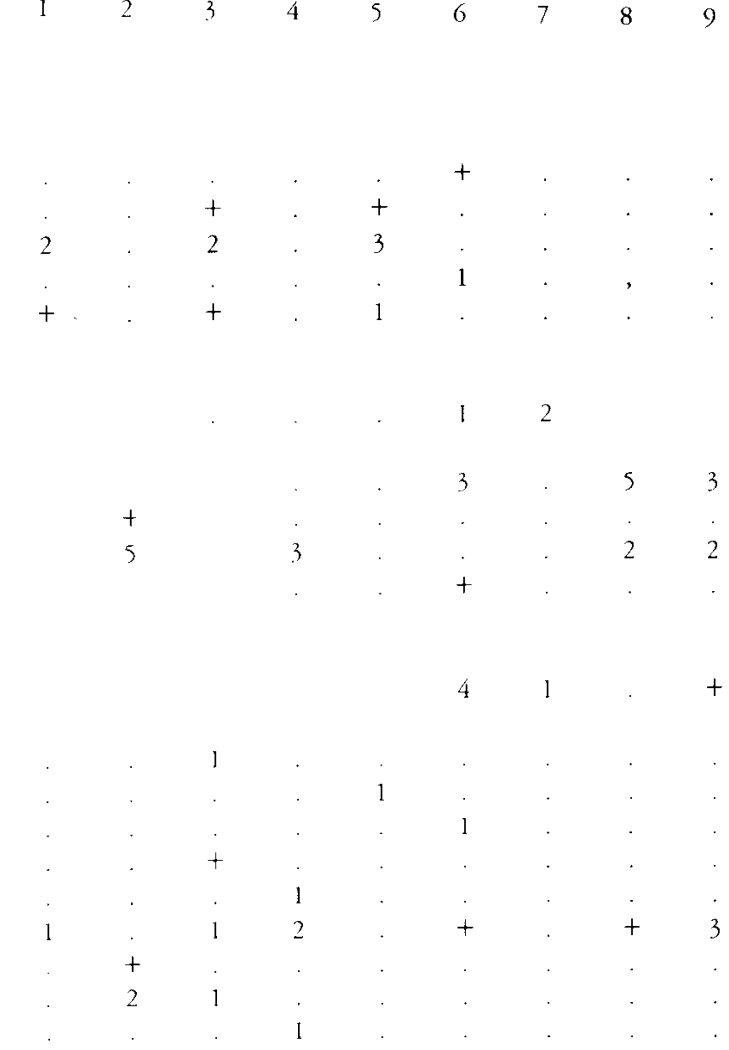

Ochthehiui quadricollis 
Tabla V - Composición del plancton de las cubetas estudiadas en Abril de 1981 Se indica el número de células por mililitro. Para cada especie el valor superior correspondiente a superficie y el inferior a profundidad (filamentos por mililicro).

Plankton from the rock pools studied in April of 1981 Data in cells/ml. The first value indicates surface concentration, rhe second une indicates depth concentration (filaments $/ \mathrm{ml}$.)

\begin{tabular}{|c|c|c|c|c|c|c|c|c|c|c|}
\hline & 1 & 2 & 3 & 4 & 5 & 6 & 7 & 8 & 9 & 10 \\
\hline \multicolumn{11}{|l|}{ Cyanophyceae } \\
\hline Chroococcus mintmus & & & & & & & & & & 13 \\
\hline Chroucocus ip. & & . & 8 & 311 & & . & & & . & . \\
\hline Aphanocapsu littoralis & $\begin{array}{l}133 \\
355\end{array}$ & $\begin{array}{l}32 \\
1349\end{array}$ & $\begin{array}{l}89 \\
188\end{array}$ & 9788 & 47 & 38280 & . & & $\begin{array}{l}73703 \\
88438\end{array}$ & 18 \\
\hline Microcoleus lyngbyaceus & 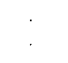 & 19 & & . & . & . & $\cdot$ & $\cdot$ & $\cdot$ & . \\
\hline Schizetbirx calcicola & $\begin{array}{c}800 \\
1000\end{array}$ & $\begin{array}{c}881 \\
15\end{array}$ & $\begin{array}{l}220 \\
500\end{array}$ & 1500 & 160 & $\begin{array}{c}3800 \\
341000\end{array}$ & $\begin{array}{c}400 \\
59000\end{array}$ & 200 & 400 & $\begin{array}{c}1200 \\
100\end{array}$ \\
\hline \multicolumn{11}{|l|}{ Dinophyceae } \\
\hline Oxyrghis murrna & : & $\begin{array}{c}1828 \\
325\end{array}$ & & & & . & 24284 & . & . & . \\
\hline \multicolumn{11}{|l|}{ Haptophyceae } \\
\hline Promnesum parium & & & & . & & . & $\begin{array}{l}13 \\
39\end{array}$ & . & & . \\
\hline $\begin{array}{l}\text { Chrysophyceae } \\
\text { Ocbromonas ip. }\end{array}$ & $\begin{array}{l}1321 \\
1985\end{array}$ & . & $\begin{array}{l}244 \\
1357\end{array}$ & 311 & 106 & $\begin{array}{l}621 \\
166\end{array}$ & $\begin{array}{l}2939 \\
152357\end{array}$ & $\begin{array}{c}42 \\
65832\end{array}$ & 2220 & 106 \\
\hline Bacillariophycese & $\begin{array}{l}727 \\
500\end{array}$ & $\begin{array}{l}32 \\
27\end{array}$ & $\begin{array}{l}467 \\
24\end{array}$ & . & & & . & 8 & & 13 \\
\hline $\begin{array}{l}\text { Euglenophuceat } \\
\text { Iimslenasp. }\end{array}$ & 13 & & 5735 & 932 & . & 8155 & . & $\dot{.}$ & $\begin{array}{l}2664 \\
2,338\end{array}$ & . \\
\hline $\begin{array}{l}\text { Prasinophyceale } \\
\text { Anteromonas gracilis }\end{array}$ & . & & 22 & & & & & & & \\
\hline Pyramimestios amplifera & & 16 & . & & & & & & & \\
\hline Pyramimonas retesiliatu & & & & 1186 & . & 166 & $\begin{array}{c}4 \\
180\end{array}$ & & & . \\
\hline Totracielmis fonmandi & & $\begin{array}{l}8066 \\
2112\end{array}$ & & 78 & & $\begin{array}{l}682 \\
499\end{array}$ & & & 888 & \\
\hline \multicolumn{11}{|l|}{ Chlorophycede } \\
\hline Drendictlat salinas & . & & & & & & 23 & . & . & : \\
\hline Brachiomenas whomatina & 868 & 241 & 133 & $\begin{array}{c}15483 \\
311\end{array}$ & 106 & $\begin{array}{r}141828 \\
67406\end{array}$ & 6 & $\begin{array}{l}2597 \\
11935\end{array}$ & $\begin{array}{l}155398 \\
103243\end{array}$ & $\begin{array}{l}567 \\
195\end{array}$ \\
\hline Curteria multifilas & 1263 & 16 & & 24665 & 1069 & 27310 & . & 1025 & . & 657 \\
\hline Chlamydtmonar ipp. & & & & $\begin{array}{c}81385 \\
1864\end{array}$ & $\begin{array}{l}7991 \\
1400\end{array}$ & & . & 319 & $\begin{array}{l}527023 \\
481150\end{array}$ & $\begin{array}{l}6467 \\
34451\end{array}$ \\
\hline Spiowerdlopsir ip. & & 160 & 23 & 777 & $\therefore$ & $\begin{array}{c}18621 \\
166\end{array}$ & 180 & . & 6660 & \\
\hline Pallowma up & & & & 311 & 364 & & & . & & \\
\hline Atenoruplidiam ip. & $\begin{array}{c}183 \\
13\end{array}$ & & 22 & & 12 & & & 8 & 390 & $\begin{array}{l}283 \\
797\end{array}$ \\
\hline Ulotbrix subluccidu & 614 & & . & . & & & . & . & & . \\
\hline Amocbicla & & & & & 106 & & & . & & \\
\hline Ciliatea & & $\begin{array}{l}32 \\
26\end{array}$ & 165 & 9)32 & & & & . & 444 & 335 \\
\hline
\end{tabular}

poco aparente. Posee dos vacuolas anteriores. El halo translúcido es ovalado o casi circular, en la mayoría de los casos con una pequeña punta en la parte posterior.

El tamaño es de 20-25 ×23-30 $\mu \mathrm{m}$ halo incluido, siendo la célula sola de 15-19x17-24 $\mu \mathrm{m}$. Los flagelos tienen una longitud de 1,25 veces el cuerpo. 


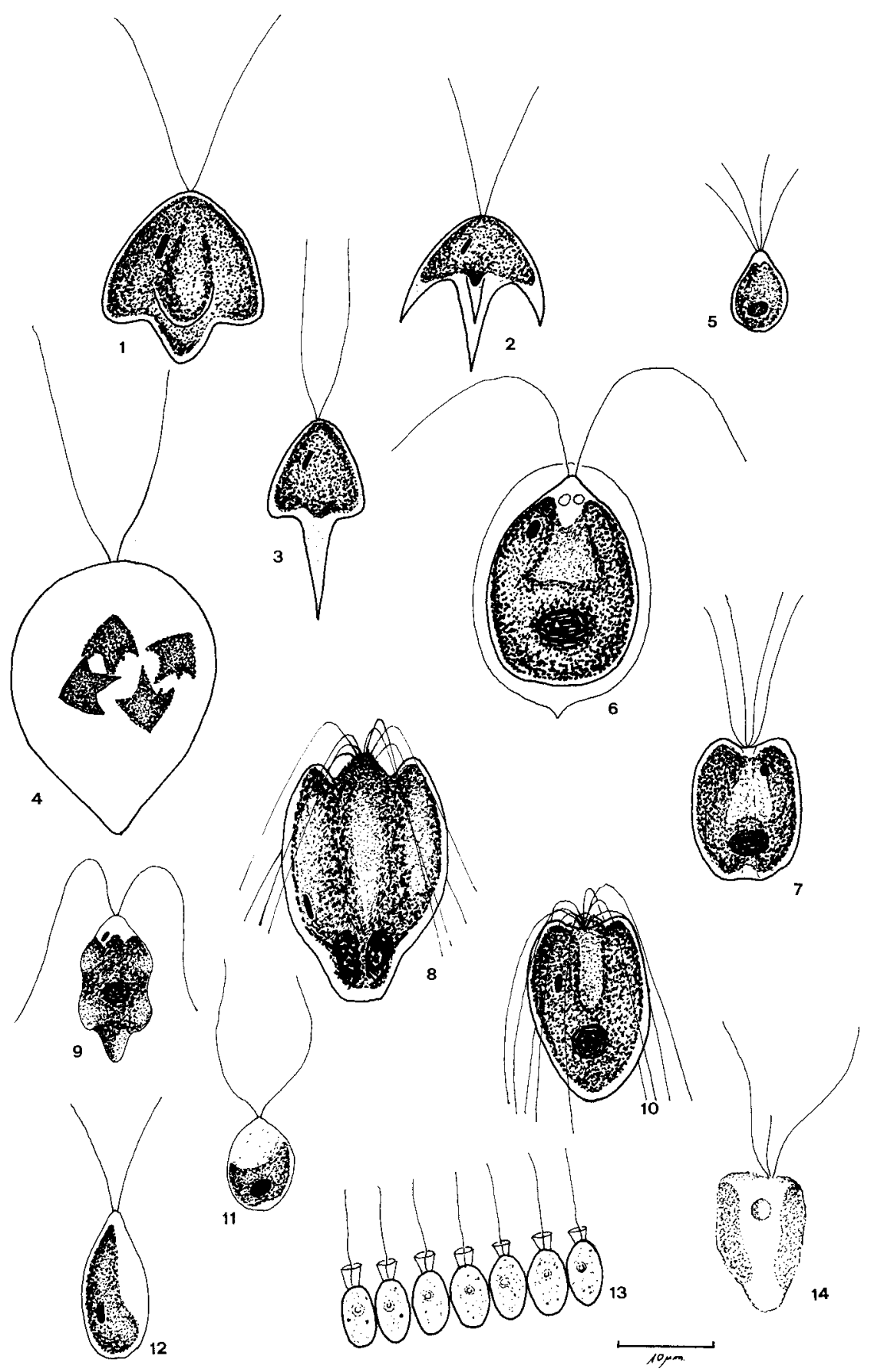

Figura 1.- Represcntación de algunos flagelados planciónicos encontrados en las cubetas supralitorales

Some flagrellates found in the rock pools

1. 2. 3, distintas formas de Brachiomonas submarina. 4, estadio de división (autósporas) de R. rabmarina. 5, Carteria multifilis 6. Sphatellopsis ip. 7. Tetraselmis fontiana. 8. Prammonas amglifera 9, Aiteromonas gracilis. 10, Puramimonas octociliata

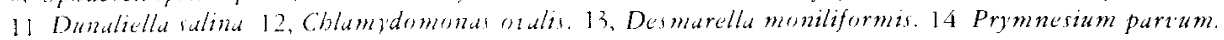


En la Tabla V se indica el número de células en las cubetas en abril de 1981. En la mayoría de ellas, los organismos se concentran en el fondo, donde las condiciones, especialmente las osmóticas, son más similares a las anteriores a la lluvia. En días soleados, a la misma hora ocupan niveles superiores.

En el zooplancton son comunes diversos ciliados, especialmente Cyclidium. Se ha observado también la presencia del coanoflagelado Desmarella moniliformis, especie ya indicada por Chrétiennot (1971), en las cubetas de la región de Marsella, donde es muy abundante y llega a formar poblaciones de hasta 27.000 células por mililitro; nosotros no hemos encontrado más de 200 (febrero de 1983).

\section{3.- Bentos}

E1 bentos de las cubetas supralitorales estudiadas era particularmente pobre. Florísticamente abundaban las diatomeas, algunos cianófitos (Entopbysalis deusta, Porphyrosipbon notarisii. Schizothrix calcicola. Spirulina subsalsa. Calothrix crustacea) y clorófitos eurihalinos (Enteromorpba intestinalis. Cladophora liniformis. Cladophora albida. Chaetomorpha y Ulothrix).

Enteromorpha intestinalis suele desprenderse y formar masas flotantes considerables. Se ha observado menores concentraciones de fitoplancton en las cubetas con abundancia de Enteromorpha. Ballesteros (1982) ofrece una extensa lista de las especies de algas bentónicas halladas en cubetas similares.

El zoobentos es también escaso, abundando nemátodos, ostrácodos y larvas de quironómidos. Las larvas de Ochtebius pueden ser abundantes y los adultos, aunque más comunes en el neuston, no faltan tampoco. El harpacticoide Trigriopus brevicornis, es propio también de estas cubetas, y suele apoyarse en el bentos.

\section{DISCUSION}

Dadas las características del muestreo sería imprudente realizar excesivas generalizaciones sobre las particularidades de las cubetas supralitorales. Los datos que se aportan, sin embargo, son suficientes para establecer un marco de cuestiones y puntos de interés.

Un estudio intenso meramente florístico y/o faunístico permitiría caracterizar mejor el poblamiento de estas cubetas, distinto del de las lagunas litorales (Comín, 1981) y, obviamente, al del mar. Los poblamientos hallados por nosotros no difieren, en líneas generales, de aquellos encontrados por Feldmann (1937) y Margalef (1949). Un estudio más prolongado probablemente aportaría una riqueza de especies comparable a la de las cubetas estudiadas por Chrétiennot (1971). La correspondencia establecida por Feldmann (1937)entre características físicas y determinadas especies no se confirma en nuestras observaciones. No hay un gradiente de salinidad entre las cubetas donde predominan Tetraselmis fontiana, Brachiomonas submarina $y$ Asteromonas gracilis, respectivamente. La tipificación de las cubetas no parece tan simple y requiere mayor estudio.

El hecho de que los organismos de estas cubetas persistan en un medio tan variable, sobre todo en salinidad y temperatura, supone la existencia de sistemas reguladores bien desarrollados. El estudio fisiológico de cualquiera de los organismos puede revelar fenómenos interesantes. A modo de ejemplo, pueden citarse los estudios con Dunaliella (Wegmann, 1971; Borowitzka y Brown, 1974; Ben-Amotx, 1975) y Monocbrysis (Graigie, 1969), que emplean glicerol y ciclohexanetetrol, respectivamente, como compuesto regulador; los realizados sobre eurihalinidad de diversas especies por Droop (1958);o estudios sobre los efectos de la temperatura (Ukeles, 1961). Se conoce poco sobre la fisiología de los organismos más comunes (Brachiomonas submarina, Tetraselmis fontiana, ...).

Otro enfoque lo plantea la elevada concentración de nutrientes y detritos. Se ha visto que Hemiselmis irescens, alga propia de estos ambientes, puede asimilar amonio y urea, en lugar de nitratos y nitritros (Antia et al.. 1975). También se ha mencionado un posible efecto fertilizante de los detritos de algas y fanerógamas marinas, arrojadas por el mar a la costa, sobre el crecimiento de las algas de las cubetas (Harrison, 1978).

La formación de películas y espumas superficiales es otro punto de interés. Es conocido el efecto dañino de la radiación ultravioleta sobre los organismos, especialmente sobre los de cutículas finas. La existencia normalmente de pocos organismos en la superficie de las aguas parece, sin embargo, ser más resultado del rápido agotamiento de nutrientes en una capa de tan poco grosor, que de los efectos negativos de aquella. En aguas ricas, donde hay una rápida 
difusión desde la parte pelágica a la superficie, el crecimiento o acumulación de organismos fotosintetizadores en la película puede ser exuberante, como ocurre en las cubetas estudiadas por nosotros.

Con enfoque ecológico, sería de interés un estudio intensivo de las fluctuaciones diarias, durante un período aceptable y bajo diversas circustancias; así como un seguimiento anual, a fin de valorar debidamente la dinámica de las cubetas supralitorales.

Dada la escasa producción primaria de la zona supralitoral rocosa, en su mayor parte integrada por líquenes, tendría interés poseer datos sobre este tipo de cubetas, las cuales deben contribuir a dicha producción, sin duda, con un elevado porcentaje.

\section{AGRADECIMIENTOS}

A Carlos Montes por la determinación de Ocbibebiur quadricollis.

\section{BIBLIOGRAFIA}

Antia, N.J.; Berland, B.R.: Bonin, D.J. \& Maestrini. S.Y. 1975. Comparative evaluation of certarn organca and inorganic sources of nitrogen for phototrophic growth of marine microalgate J mar biol Ass U K 55 519.539

Ballesteros, E. 1982. Primer intento de tipificación de la vegetat cion marina y liroral sobre sustrato rocoso de la CostaB Brava Oecol Aq $6: 163.173$

Ballesteros, E. 1983. Les zones supralitorals I mediolitorals de les Illes Medes, 2n J.D Ros. J M (Gili 1 I Olivella Ld Els sistemes naturals de les Illes Medes Institut d'Estudis Catalans Barcelona Ben-A motz, A. 1975. Adaptation of rhe unicellular alga Dumaliellat purta to al saline environment J Phycol 11 50-54

Borowitzka, L.J. \& Brown, A.D., 1974́. The salt relutions of marine and halophilic species of the unicellular green algace Dunaliclla The role of phlycerol as a compatible solute Arch Microbiol 96: 37.52

Bourrelly. P. 1958. Algues micruscopiques de quelques curettes supralittorales de la region de Dinard Vrrh Int Ver Limnol 13 $683-686$

Chretiennot, M.J.. 1971. Ltude systemattque et evolution des populations nanoplanctoniques des flaques supraliterales de lat region marseillaise These Doctorar Université d' Aix. Marseille? Comin, F., 1981. Limnología comparada de las lagunas costeras del Delta del Ebro. Tesis Doctoral Universidad de Barcelona Graigie, J.S., 1969. Some salinity induced changes in growth, pigments and cyclohexanetetrol content of Monochrysis lutberi. J Fish Res Bd. Can 26. 2959-67

Droop, M.R., 1953. On the ecology of flagellates from some brackish and freshwater rock pools in Finland. Acr Bot Fenn 51 3.52

Droop, M.R., 1958. Optimum, relative and actual ionic concentracons for growth of some euryhaline algae Verh. Int Ver Limnol. I 3: $722 \cdot 30$

Feldmann, J., 1937. Recherches sur la végetation rnarine de la Méditerrenée: La Cote des Alberes, Wolf. Rouen

Harrison, P.G., 1978. Growth of Ulia fenestrata (Chlorophyta) microcosm rich in Zostera marnal (Anthophyta) detritus J Phycol. 14: $100-103$

Huber-Pestalozzi, G., 1961. Das Phytoplankton des Susswassers Schweizerbart'sche Verlagsuchhandlung Sturtgart. 5 Chloro phyceae. Ordung: Volvocales.

Margalef, R.; Planas, D.; Armengol, J.; Vidal, A.; Prat, N; Acde: martae. Publ. Inst Biol Apl 6:83-102

Margalet, R.; Planas, D.; Armengol, J.; Vidal, A.; Prat. N.; Guiset, A.; Toja, J. \& Estrada, M. 1976. Limnología de los embalses españoles. Dirección de Obras Hidraúlicas. Publ $n^{0} 123$ Peres, J.M. \& Picard, J., 1964. Nouveau manuel de Bionomie Henrhique de la Mer Méditerranée Rec Trav. St. Mar End. 31 (47): 5-137

Schwoerbel, J., 1975. Métodos de Hidrobiologia Ed Blume. Madrid

Talling, J.F. \& Driver, D.. 1963. Some problems in rhe estumatwon of chlorophyll in phytoplankton Proceedings Conterence of Primary Productivity Measurement, Marine and Freshwater Hawaii 1961 US Atomic Energy Comm TID-7633: 142-146 Ukeles. R.. 1961. The effect of temperature on rhr growth and survival of several marıne algal species Biol Bull 120: 255-264 Vacelet, E.. 1959. Etude physico-chimique des flaques suprali terales a solinité variable Relations avec les peuplements Ree Trav Sr Mar End 29)(17) 5-88

Vacelet, E.. 1969. Role des populations phytoplanctoniques et bacteriennes dans le cycle du phosphore et de l'azoteen meret dans les flaques supralietorales dugulfe de Marsaitle Téthys I ( 1 l l-105 Vollenweider. R.A., 1969. A manual on Methods for measuring Promary Producrion in Aquatic Environments Balckwell. Oxford Wegmann, K.. 1971. Osmotic regulation of photosynthetic glycerol production in Dundiella. Biochem Biophys Acta. 214 $317-323$ 